\title{
Universal Attractors of Reversible Aggregate-Reorganization Processes
}

\author{
Stefan Großkinsky ${ }^{1}$, Marc Timme ${ }^{2}$, and Björn Naundorf ${ }^{2}$ \\ ${ }^{1}$ Zentrum Mathematik, TU München, 80290 München, Germany \\ ${ }^{2}$ Max-Planck-Institut für Strömungsforschung, 37073 Göttingen, Germany
}

\begin{abstract}
We analyze a general class of reversible aggregate-reorganization processes. These processes are shown to exhibit globally attracting equilibrium distributions, which are universal, i.e. identical for large classes of models. Furthermore, the analysis implies that for studies of equilibrium properties of any such process, computationally expensive reorganization dynamics such as random walks can be replaced by more efficient, yet simpler methods. As a particular application, our results explain the recent observation of the formation of similar fractal aggregates from different initial structures by diffusive reorganization [Filoche and Sapoval, Phys. Rev. Lett. 85, 5118 (2000)].

PACS numbers: $61.43 . \mathrm{Hv}, 64.60 . \mathrm{Cn}$, 89.75.Fb
\end{abstract}

Many complex systems like microbial colonies, colloidal aggregates, or particle-adsorbate structures are composed of a large number of similar units |1]|. For the growths of such particle clusters, the model of diffusionlimited aggregation (DLA) has been introduced |2 and intensively investigated in several generalizations [1, 3, 4. 4 . In these non-equilibrium models, particles are permanently injected into the system and stick irreversibly to an existing cluster which grows towards a fractal structure. In real systems, however, particles are often rearranged, dynamically transforming the aggregate structure. This rearrangement is appropriately modelled by processes exhibiting reversible dynamics [1, 3, 伆.

Recently, Filoche and Sapoval have proposed a specific model of diffusion-reorganized aggregation (DRA) [5] in which particles detach from the boundary of a given cluster of fixed size and reaggregate after a random walk on the underlying square lattice. In numerical simulations, they observe the formation of fractal structures similar to those found in various irreversible models. The DRA process transforms different initial aggregates, e.g. simple one-dimensional and two-dimensional structures, into statistically similar fractal aggregates after many reorganization steps.

In this Letter, we analyze a general class of reversible processes that dynamically reorganize a fixed number of particles constituting one connected aggregate on some lattice. Particles are allowed to reorganize by any reversible process that retains a connected aggregate, including diffusion as a particular case. We prove that all these processes exhibit equilibrium distributions that are globally attracting. Our general mathematical perspective reveals that such attractors are universal, i.e. independent of the dynamical model details: In discrete-time models, this distribution only depends on the mechanism of particle disaggregation but is independent of the subsequent particle repositioning dynamics. In particular, this implies that ordinary diffusion and different mechanisms like surface diffusion [1] or Lévy-flights $\| 6]$ are equivalent in this context. Furthermore, we show that in continuous-time models equilibria are independent of both the mechanism of repositioning and that of disaggregation. On a $d$-dimensional cubic lattice, if all connected aggregates are accessible by the repositioning dynamics, this leads to the uniform distribution that is known as the ensemble of lattice animals (cf. 怆, 8, 91), currently also investigated in number theory under the name polyominoes (\|10] and refs. therein).

In addition to these analytical results, we present strong numerical evidence that, for sufficiently large aggregates, there is no significant difference between equilibrium distributions obtained from discrete- or continuous-time models. Our results are valid for a general class of processes, including reorganization of aggregates consisting of different kinds of particles as well as repositioning of whole clusters instead of single particles. Here, for simplicity of presentation, we focus on processes in which single, identical particles are reorganized.

We consider reorganization processes of aggregates certain connected collections of a fixed number $N$ of particles on some lattice. Starting from an aggregate $A$, one step of the discrete-time dynamics is defined by the following two rules (Fig. 1):

(i) Randomly select a particle from a set of free particles $F(A)$ with equal probability, where $F(A)$ is chosen such that the aggregate remains connected.

(ii) Reposition this particle by a reversible process at some site $x$ of possible aggregation sites $D(A, B)$ of an intermediate object. By reversibility, this particle is again a free particle of the new aggregate $B$.

The above two rules are repeatedly applied to the evolving aggregate. Note that physically the step-by-step application of the two rules reflects a separation of time scales that is realized in many processes [1]: The disaggregation of a particle occurs much slower than its repositioning. To model a specific reorganization process, the sets $F(A)$ of free particles (i), the repositioning (ii) as well as the underlying lattice can be defined as desired 


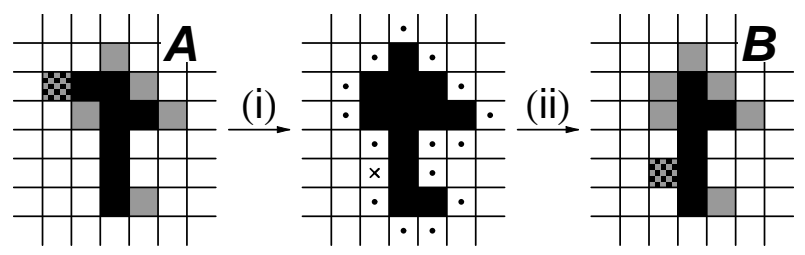

Figure 1: Model of a specific reversible aggregatereorganization process: One particle (checkered) is selected from a set of free particles $F(A)$ (gray) of aggregate $A$ according to rule (i) and detaches from the aggregate. By a reversible process, e.g. diffusion, it is repositioned at one $(\times)$ of the free aggregation sites $D(A, B)(\bullet)$ of the intermediate object according to rule (ii). Reversibility requires that the deposited particle (checkered) is again a free particle in $F(B)$ (gray) and may be selected in the next reorganization step.

as long as every aggregate under consideration can be reached iterating the above two rules.

To be specific we focus on $d$-dimensional cubic lattices, but the analysis can be analogously applied for arbitrary lattices. We fix aggregates on a lattice of $(N+1)^{d}$ sites with periodic boundary conditions and view translated aggregates of the same shape as different states. A given process is identified as a Markov chain [11] with state space $S_{N}$ consisting of all connected aggregates of $N$ particles, which have at least one free particle, i.e. non-empty $F(A)$. The state of this discrete-time process is then described by the distribution $p_{t}(A)$, giving the probability that after $t$ reorganization steps the aggregate $A$ is present. Starting with initial distribution $p_{0}(A)$, a transition matrix $T$ specifies the time evolution

$$
\mathbf{p}_{t}=T \mathbf{p}_{t-1}=T^{t} \mathbf{p}_{0}
$$

of the state vector $\mathbf{p}_{t}:=\left(p_{t}\left(A_{1}\right), \ldots, p_{t}\left(A_{\left|S_{N}\right|}\right)\right)^{\mathrm{T}}$. As $S_{N}$ is finite, the long-time $(t \rightarrow \infty)$ behaviour of the discretetime Markov chain is determined by a steady state distribution $\mathbf{p}_{d}^{*}=T \mathbf{p}_{d}^{*}$. If the chain is irreducible, i.e. every aggregate $A \in S_{N}$ can be reached by the process from every other, $\mathbf{p}_{d}^{*}$ is the global attractor towards which every initial distribution converges [11. This uniqueness property depends on the choice of free particles $F(A)$. In general, one reasonably restricts the state space $S_{N}$ to an appropriate subset of aggregates consistent with $F(A)$. For instance, if $F(A)$ is the set of all one-bond particles [8], only tree-like aggregates are considered. In such cases our analysis applies to the respective irreducible subset of $S_{N}$ (also simply denoted by $S_{N}$ in the following). With this convention, the stationary distribution $\mathbf{p}_{d}^{*}$ is the unique attractor for every initial distribution $\mathbf{p}_{0}$ on $S_{N}$. We now specify the transition matrix $T$ which completely determines the dynamics of the process.

If a one-step transition from aggregate $A$ to $B$ is possible, denoted by $A \leftrightarrow B$, the particle which is disaggregated and its reaggregation site are determined uniquely
[12]. Thus the underlying lattice does not explicitly enter the analysis. The transition probabilities are given by the matrix elements

$$
T_{B A}= \begin{cases}\frac{1}{|F(A)|} r_{B A} & \text { if } A \leftrightarrow B \\ 0 & \text { otherwise }\end{cases}
$$

which, if non-zero, consist of two factors: According to rule (i) a free particle is randomly selected from $F(A)$ with uniform probability $1 /|F(A)|$. Then, the particle is repositioned at the intermediate aggregate with probability $r_{B A}$ to obtain aggregate $B$ according to rule (ii). The reversibility of the repositioning procedure is characterized by the symmetry $r_{B A}=r_{A B}$. Thus, the dynamics is described by the master equation

$$
\begin{aligned}
p_{t+1}(A)-p_{t}(A) & =\sum_{B \in S_{N}}\left[T_{A B} p_{t}(B)-T_{B A} p_{t}(A)\right] \\
& =\sum_{B \in S_{N}}\left[\frac{p_{t}(B)}{|F(B)|}-\frac{p_{t}(A)}{|F(A)|}\right] r_{A B} .(3)
\end{aligned}
$$

A steady state solution of this equation is obviously given by the equilibrium distribution

$$
p_{d}^{*}(A)=|F(A)| / \sum_{B \in S_{N}}|F(B)|,
$$

which fulfills the condition of detailed balance, i.e. $\frac{p_{d}^{*}(B)}{|F(B)|}=\frac{p_{d}^{*}(A)}{|F(A)|}$ for all $A, B \in S_{N}$. From the above we know that $\mathbf{p}_{d}^{*}$ is the unique attractor of the dynamics (3). Hence, in equilibrium the probability to observe an aggregate $A$ is proportional to its number $|F(A)|$ of free particles.

It seems natural to realize repositioning by a random walk of the disaggregated particle, because it models diffusion in real systems 河, 81. This random walk determines the transition probabilities $r_{A B}=r_{B A}$ between aggregates $A$ and $B$. However, independent of the actual values of $r_{A B}$, and hence the repositioning procedure (ii), the process leads to the same equilibrium distribution $\mathbf{p}_{d}^{*}$. Thus, to study the equilibrium properties of the reorganization process, one can replace the random walk in numerical simulations: For instance, after selection of a particle, it may just be repositioned at every aggregation site with equal probability $r_{A B}=1 /|D(A, B)|$, where $D(A, B)$ is the set of possible aggregation sites of the intermediate aggregate (cf. Fig. [1 and ref. $[\nabla \mid)$. This straightforward procedure is much simpler to implement than random walks and significantly reduces the computational effort. Furthermore, one expects a faster convergence to the equilibrium distribution |13|: While for diffusive repositioning the particle re-aggregates with high probability near or even at its disaggregation site, the alternative process leads to a fast spreading of particles so that the state space is likely to be sampled faster. Moreover, diffusing particles in $d \geq 2$ dimensions have an infinite average return time leading to computational 
complications [5, 81 that can be avoided using alternative processes.

Up to now, we have considered discrete-time evolution rules, which arise when performing computer simulations. However, for real reorganization processes, continuous-time modelling, where the free particles $F(A)$ disaggregate independently at a rate $\gamma$, often seems more appropriate. This leads to a continuous-time Markov chain with completely symmetric transition rates $\gamma r_{A B}$ occurring in the master equation

$$
\frac{d}{d t} p_{t}(A)=\sum_{B \in S_{N}} \gamma\left[p_{t}(B)-p_{t}(A)\right] r_{A B}
$$

Thus, the attractor of these continuous-time processes is

$$
p_{c}^{*}(A)=1 /\left|S_{N}\right| .
$$

This is a universal equilibrium distribution which is independent of all dynamical model details. It is important to note that, although $F(A)$ does not enter (6), it implicitly determines the set $S_{N}$ of accessible aggregates. In the case of $d$-dimensional cubic lattices and for every choice of $F(A)$ for which the process can reach all possible connected aggregates of $N$ particles (cf. our simulations below), this uniform distribution $\mathbf{p}_{c}^{*}$ is known as the ensemble of lattice animals [9, 10].

The question arises, how relevant the differences between discrete- and continuous-time modelling are. To answer this question, we consider the fraction

$$
f_{N}(A)=|F(A)| / N
$$

of free particles of an aggregate $A$ of $N$ particles. This observable determines the difference in the equilibrium distributions of discrete-time and their associated continuous-time models because it directly enters (4) in the form $p_{d}^{*}(A)=f_{N}(A) / \sum_{B} f_{N}(B)$. In equilibrium, the average fractions of free particles obviously satisfy the inequality

$$
\left\langle f_{N}\right\rangle_{\mathbf{p}_{d}^{*}}=\sum_{A} f_{N}(A) p_{d}^{*}(A)=\frac{\sum_{A} f_{N}^{2}(A)}{\sum_{B} f_{N}(B)} \geq\left\langle f_{N}\right\rangle_{\mathbf{p}_{c}^{*}}
$$

because $\left\langle f_{N}\right\rangle_{\mathbf{p}_{c}^{*}}=\sum_{B} f_{N}(B) /\left|S_{N}\right|$ by definition. Nevertheless, Monte-Carlo simulations of a specific process [14] show that the averages $\left\langle f_{N}\right\rangle$ clearly converge towards the same value for discrete- and continuous-time models (Fig. 2). Differences become insignificant already for moderately sized aggregates.

This is quantified by considering the widths of the equilibrium distributions $\sigma\left(f_{N}\right):=\left\langle\left(f_{N}-\left\langle f_{N}\right\rangle\right)^{2}\right\rangle^{\frac{1}{2}}$ (Fig. 2, inset). We find a power law decay $\sigma\left(f_{N}\right) \sim N^{-\alpha}$ with $\alpha=0.50 \pm 0.02$ for both time scales as would be expected for large random structures. This has important

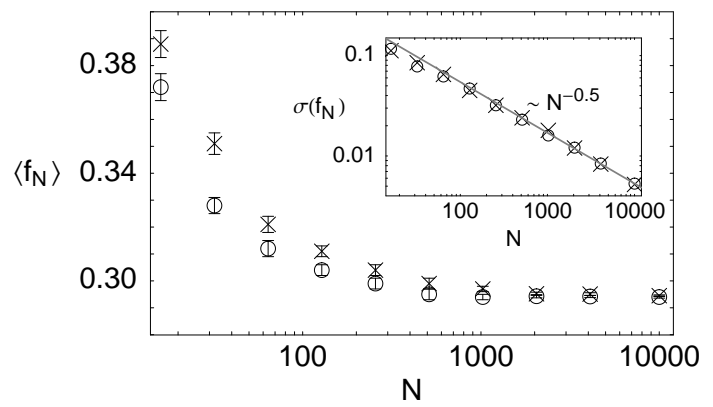

Figure 2: Comparison of average fractions $\left\langle f_{N}\right\rangle$ of free particles: discrete-time $(\times)$ and its associated continuous-time model $(\bigcirc)$. Error bars denote standard error of sample mean. Inset: Widths $\sigma\left(f_{N}\right)$ for both time scales.

consequences: Calculations similar to the above (8) show immediately that

$$
\left\langle g_{N}\right\rangle_{\mathbf{p}_{d}^{*}}=\left\langle g_{N}\right\rangle_{\mathbf{p}_{c}^{*}}\left(1+\mathcal{O}\left(N^{-\alpha}\right)\right)
$$

for every observable $g_{N}(A)$. Thus, for sufficiently large aggregates, measurements of arbitrary observables, and, equivalently, the equilibrium distributions, in discreteand continuous-time models cannot be distinguished. This reflects the fact that the number $|F(A)|$ of free particles of almost all aggregates $A \in S_{N}$ is close to its uniform ensemble average $\langle|F(A)|\rangle$ for sufficiently large $N$. Since this fact is of purely configurational origin, we expect a similar convergence (9) for other models, also in $d>2$ dimensions.

As one important observable, we have studied the fractal dimensions of aggregates from both processes. Sample aggregates as well as box-counting dimensions $\left\langle D_{N}\right\rangle$ are shown in Fig. 3. Even for moderately sized aggregates, no significant differences have been observed. With increasing aggregate sizes, the $\left\langle D_{N}\right\rangle$ approach from below [15] the well-estimated fractal dimension $D \approx 1.56$ [9, 10] of square-lattice animals.

The above results predict that the uniform distribution among all connected aggregates which can be reached by a specific process is the globally attracting, unique equilibrium distribution for a wide class of models. In particular, our results explain the recent observation that fractal aggregates are reached from arbitrary initial structures by a certain diffusive reorganization process |5|. Nevertheless, our results strongly indicate that the equilibrium aggregates from that study are in the same universality class as lattice animals and thus have a fractal dimension $D \approx 1.56$ and not, as suggested, $D=1.74 \pm 0.02$ 疖. A possible explanation of this discrepancy is that simulations in that study did not reach equilibrium at the time of measurement, despite a huge number of simulation steps. In fact, in our simulations [14 aggregates of $N=10000$ particles only reached equilibrium after about $2 \times 10^{8}$ particle rearrangements. 


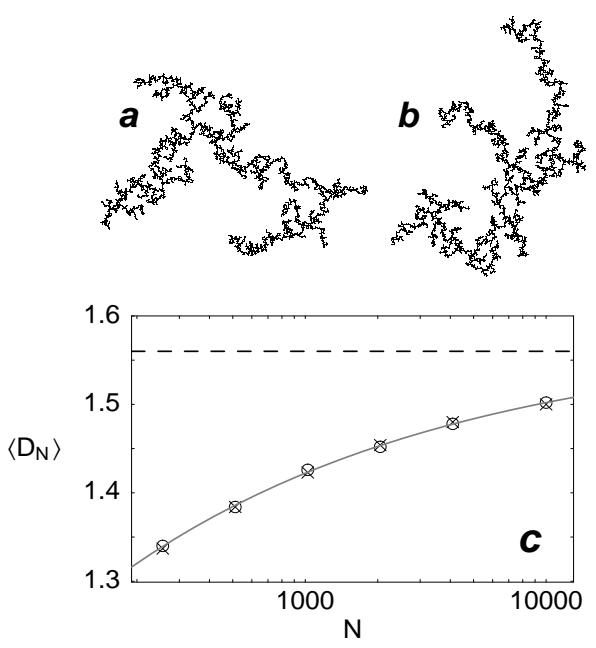

Figure 3: (a,b) Equilibrium fractal aggregates of $N=10000$ particles, started from a $100 \times 100$ square, after $10^{9}$ particle rearrangements [14]. (a) Discrete-time model, (b) associated continuous-time model. (c) Box-counting dimensions $\left\langle D_{N}\right\rangle$ for both time-scales are indistinguishable and approach from below [15] the fractal dimension $D \approx 1.56$ (dashed line) $[9,10]$.

It is important to note, that in certain systems such a slow convergence may imply that the equilibrium state is not reached during the time of real or numerical experiments (cf. [5]). Thus although an equilibrium distribution is guaranteed to be reached, the long-term behavior of such systems may be governed by transient states with long lifetimes and non-universal statistical properties.

In summary, we have shown that large classes of reversible aggregate-reorganization processes possess universal attractors which are independent of the model details but may in general depend on whether discrete- or continuous-time modelling is used. Furthermore, our numerical investigations indicate that statistical properties are essentially identical for both time scales. These results imply that commonly used diffusion modelling can be replaced by computationally fast methods which in addition lead to a faster convergence towards the (identical) equilibrium distribution. In general, one specific process of aggregate-reorganization can be investigated in a number of different alternative models. Which alternative is used can be freely chosen depending on which one is more convenient to study analytically or by computer simulations.

In particular, our results explain the recently discovered "statistical attractors" in diffusive reorganization processes [5] but predict a different final equilibrium state. More generally, the results can serve as a basis for investigating equilibrium statistical properties of a general class of aggregate-reorganization processes. Reversible reorganization is always characterized by the symmetry $r_{A B}=r_{B A}$, which, in turn, has been the main requirement for universality. In particular, aggregates consisting of different kinds $\alpha$ of particles disaggregating with particle-specific rates $\gamma_{\alpha}$ are also described because a transition $A \leftrightarrow B$ determines the disaggregating particle uniquely. Moreover, the theory may be generalized to reorganization of whole sub-clusters rather than just single particles (cf. also [3, 7]). Hence more complex models of aggregate-reorganization can also be captured.

We have imposed only a small number of requirements on the dynamics which appear to be natural assumptions in many physical situations, in particular reversibility of repositioning and separation of time-scales. Thus our main results might not only be of importance to aggregate-reorganization processes. We hope that the basic ideas can also find applications in studies of other complex systems exhibiting reversible reorganization.

We thank M. Biehl, D. Brockmann, T. Geisel, L. Hufnagel, T. Kottos, and M. Prähofer for useful discussions. This work has been supported by the Max Planck Society.

[1] A. Bunde and S. Havlin (eds.), Fractals and Disordered Systems, (Springer, Berlin, 1991); T. Vicsek, Fractal Growth Phenomena (World Scientific, Singapore, 1992); P. Meakin, Fractals, scaling and growth far from equilibrium (Cambridge Univ. Press, Cambridge, 1998).

[2] T.A. Witten and L.M. Sander, Phys. Rev. Lett. 47, 1400 (1981).

[3] P. Meakin, in Phase Transitions and Critical Phenomena, Vol. 12, edited by C. Domb and J.L. Lebowitz (Academic Press, London, 1988).

[4] A. Erzan, L. Pietronero, and A. Vespignani, Rev. Mod. Phys. 67, 545 (1995).

[5] M. Filoche and B. Sapoval, Phys. Rev. Lett. 85, 5118 (2000).

[6] P. Meakin, Phys. Rev. B 29, 3722 (1984).

[7] E.J. Janse van Rensburg and N. Madras, J. Phys. A 25, 303 (1992).

[8] R. Botet and R. Jullien, Phys. Rev. Lett. 55, 1943 (1985); R. Wessel and R.C. Ball, Phys. Rev. A 45, R2177 (1992).

[9] T.C. Lubensky and J. Isaacson, Phys. Rev. A 20, 2130 (1979); A. L. Stella et al., Phys. Rev. Lett. 69, 3650 (1992); D. Stauffer and A. Aharony, Introduction to Percolation Theory (Taylor and Francis, London, 1994).

[10] S. Golomb, Polyominoes: Puzzles, Patterns, Problems, and Packings, 2nd ed. (Princeton Univ. Press, Princeton, 1994); I. Jensen and A.J. Guttmann, J. Phys. A 33, L257 (2000).

[11] J.R. Norris, Markov chains, (Cambridge Univ. Press, Cambridge, 1997).

[12] Uniqueness is guaranteed only if $B \neq A$. If $B=A$, any particle from $F(A)$ can disaggregate and must then reaggregate at the same site. This is easily included into the definition of $r_{A A}$.

[13] V. Guruswami, Rapidly Mixing Markov Chains, Survey (MIT Laboratory for Computer Science, Cambridge, 2000).

[14] Monte-Carlo simulations: square lattice, $F(A)=\{$ all particles which, if removed, keep the aggregate connected $\}$. 
Expectation values estimated from at least 250 equilibrium realizations of aggregates (depending on $N$ ).

[15] Finite size scaling $D_{\infty}-\left\langle D_{N}\right\rangle \sim N^{-\nu}$ with $\nu=0.33 \pm 0.04$ leads to a dimension estimate of $D_{\infty}=1.56 \pm 0.02$. 